\title{
STUDIO PRELIMINARE SULLE OSCILLAZIONI LIBERE DEL IAAGO DI IDRO
}

\author{
Maria Cegitia Spadea
}

Dal lavoro "I laghi d'halia" di R. Riccardi, si traggono le seguenti notizic circa $\mathrm{i}$ dati che caratterizzano il lago di Idro: lat. $45^{\circ} 47^{\prime}$ N circa; Long. 1"17' W a. (Monte Mario); alı. nu 368; sup. kmq 10,87; lungh. mass. fra grli estremi $\mathrm{km}$ 10, lungo la linea di valle $\mathrm{km} 10,8$; mass. largh. km 2; mass. prof. m. 122.

Questi ed altri dati vamo considerati come approssimativi, essendo sogrgetti a tante cause di variazione: diversiti di metodi di misura, cambiamenti stagionali o secolari o accidentali, ece.

Per la determinazione analitica delle fondamentali caratieristiche dei moti liberi del lago, si i fatlo ricorso alla carta hatimetrica del lago stesso publblicala dal De Arostini nell'a Atlante dei laghi Italiani ". La carla $i$ stata riportala al 25.000 .

Il lago i stato diviso, lungo la linea di valle, in 32 sezioni di 300 in $300 \mathrm{~m}$ salvo le prime due, per le quali l'erpuidistanza è stata di $150 \mathrm{~m}$. Detle sezioni vanno contate a partire dall'estremo Nord (figrg. 1-2).

La larwhezza $b(x)$ delle singole sezioni in superficie, l'area $S(x)$ delle sezioni stesse. l'area $v(x)$ della superficie libera del lagro fra due sezioni consecutive, l'area $V(x)$ fra le varie sezioni e l'estremo Nord del lagro e $\mathrm{i}$ valori della funzione $n(x)$ (uguale al prodotto $S(x) \cdot b(x)$ ) per le 32 sezioni, sono riportati nella tabella $I$.

Si i proveduto quindi alla determinazione della curra nornale del lago, che come $:$ noto, si ottienc rappresentando $\sigma(x)$ in funzione di $V(x)$. La figura 3 ne da l'andamento.

La complessita della curra normale ha portato ad escludere l'applicazione di uno dei metodi suggeriti da Chrystal, per la determinazione degli elementi caratleristici delle sesse del lago.

Si i fallo pertanto ricorso ai melodi di Hidaka e di Defant, l'applicazione dei quali prescinde dalla forma della curva normale.

Il metodo di IIidaka, la cui teoria è stata esposta altrove, è stato applicalo per il caso $m=2$. 


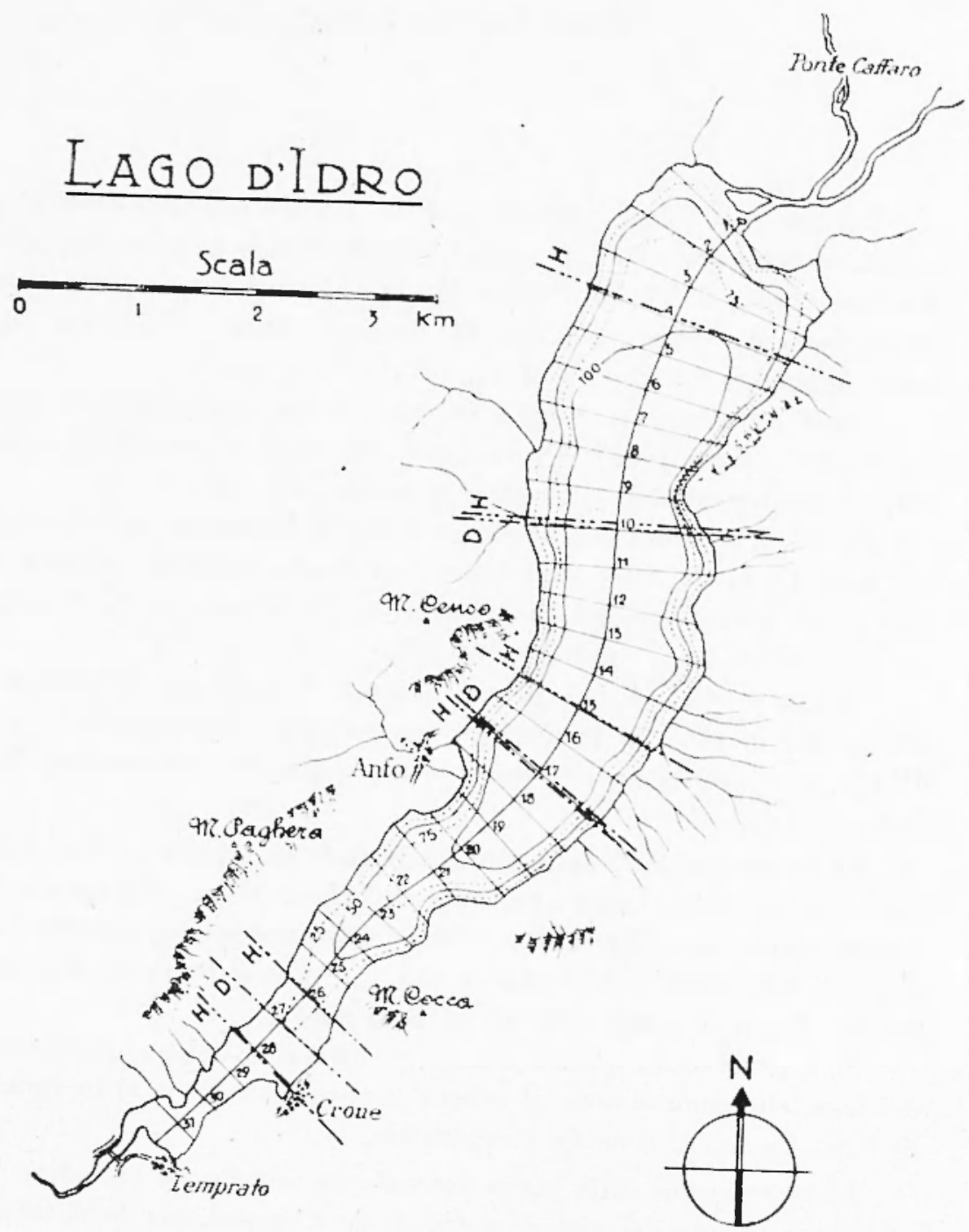

Fig. 1 
Tabella I

\begin{tabular}{|c|c|c|c|c|c|c|c|c|c|c|c|c|c|c|}
\hline . & ${ }_{\mathrm{n}}(\mathrm{x})$ & $\begin{array}{c}S(x) \\
10^{3} \text { I1. }\end{array}$ & $\begin{array}{c}\text { lararh. } \\
b(x) \\
\text { min }\end{array}$ & $\begin{array}{l}r() \\
\ln 11,2\end{array}$ & $\begin{array}{l}V(\mathrm{x}) \\
\text { hin. }\end{array}$ & $\begin{array}{c}\sigma(x) \\
{[h n, 3]}\end{array}$ & $z=\frac{\mathbf{J}}{\boldsymbol{a}}$ & $\frac{z^{2}(1-z)^{2}}{\sigma(1)}$ & $\Delta z$ & 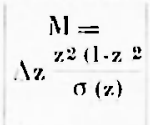 & $11 \%$ & $M: 2$ & N $\%$ & $\mathbf{M} \boldsymbol{x}^{\prime}$ \\
\hline 0 & 0 & 0 & 0 & 0 & 0 & 0 & 0 & 0 & 0 & & & & & \\
\hline 1 & 150 & 29.75 & $1+12$ & 22,297 & 22,297 & $12, ' 07$ & $.0 \quad 993$ & 9,284 & .01993 & $\cdot 18503$ & .00369 & $\cdot 00007$ & $\cdot 000002$ & .00000004 \\
\hline 2 & 300 & 96,25 & 1825 & $48,6+19$ & $70.9 \cdot 46$ & 175,656 & $\cdot 06341$ & 20.096 & $\cdot 0.43 .48$ & .87377 & .05511 & .00351 & .00022 & .00002 \\
\hline 3 & s & 133,00 & 1875 & 57,432 & 128,378 & $2.49,375$ & .11473 & $+1,3+3$ & $.0513:$ & 2,12172 & $\cdot 2+13.12$ & $\cdot 02792$ & $\cdot 00320$ & $\cdot 00036$ \\
\hline 4 & - & $1+3,50$ & 1775 & 58,108 & 186,186 & $2^{-} 4,712$ & $\cdot 16667$ & 75,733 & .05191 & 3,93357 & .65561 & $\cdot 10927$ & $.0182 \mathrm{~L}$ & .00303 \\
\hline 5 & , & 149,00 & 1775 & 54,054 & $2 .+1,5+10$ & $26 \cdot 4,475$ & $\cdot 21.197$ & 107,685 & .01830 & 5,20119 & 1,11810 & .2 .1035 & $\cdot 0516.5$ & .01113 \\
\hline 6 & $\bullet$ & 151,25 & 1675 & 57,432 & 297,972 & $253,34.4$ & $\cdot 266330$ & 150,704 & .05133 & 7,73564 & 2,06000 & .54861 & $\cdot 1+613$ & $\cdot 03891$ \\
\hline 7 & s & $1.11,75$ & $16 \geq 0$ & 18,619 & 316,621 & 229,635 & $\cdot 30973$ & 199,055 & $\cdot 01348$ & $8,65.191$ & 2,68112 & .83053 & 31 & .07971 \\
\hline 8 & s & 117,75 & 1325 & 41,892 & 388,513 & $156,1: 19$ & $\cdot 34 \quad 22$ & 329,255 & .037 .4 .4 & 12,32731 & 1,28029 & 1,48618 & .51602 & $\cdot 17912$ \\
\hline 9 & . & 118,25 & 1320 & $43,2+3$ & 431.756 & 156,8990 & $\because 8587$ & 359.792 & $\cdot 03865$ & 13,90596 & 5,36589 & 1,06070 & .79904 & .30830 \\
\hline 10 & s & 111,75 & 1515 & $+1,622$ & $478,3=8$ & 169,31 & $\cdot 427 \div 4$ & $35 ?, 808$ & .04167 & $11,7+318$ & 0,30330 & $2,69.191$ & 1.1 & $\cdot 19257$ \\
\hline 11 & , & 137,25 & 1475 & $48,6+9$ & 527,027 & 202,441 & $.471^{111}$ & 306,153 & $\cdot 0.4 \cdot 47$ & $13,3.3021$ & 6.27866 & 2,95731 & 1,39287 & .65611 \\
\hline 12 & , & 120,50 & 1340 & 47,297 & $57.1,32.4$ & 161,40 & $\cdot 51328$ & 386,511 & $\cdot 0.4227$ & 16,33782 & 8,38588 & 4,30136 & 2,20936 & 1,13401 \\
\hline 13 & , & 142,25 & 1.163 & +1.892 & 016,216 & $2: 8,395$ & .55072 & 293,768 & .0 .374 .1 & 10,99867 & 6,05719 & 3,33579 & 1,83711 & 1,01166 \\
\hline 1.1 & - & 127,$2 ;$ & 1375 & $4.4,595$ & 6601,811 & 174,969 & $.590: 8$ & 334,116 & $\cdot 03^{\prime}, 86$ & 13,31786 & 7,86526 & 1,67500 & 2,74321 & 1,62012 \\
\hline 15 & , & 117.25 & 1275 & 41,216 & 702,027 & $1.49,194$ & .62742 & 365.566 & $.036^{-} 4$ & 13.43089 & 8,42681 & 5,28720 & 3,31730 & 2,08138 \\
\hline 16 & , & 119,0 & 1320 & 41), 510 & 712,567 & 157,030 & .66365 & 317,227 & -03623 & 11,49313 & $7,627.42$ & 5,06192 & 3,35933 & 2,22 \\
\hline 17 & , & 129,75 & 1350 & 17,973 & $790,5+40$ & 175,162 & $\cdot 706: 2$ & $245,4^{\prime} 0$ & $\cdot 0.4 \cdot 87$ & 10,52158 & 7,43371 & 06 & 3,71065 & 2,62166 \\
\hline 18 & , & 107,00 & 1320 & $13,2,43$ & 833,783 & $141,24)$ & $\cdot 74517$ & 255,310 & $\cdot 03865$ & 9,86773 & $7,3531+4$ & $5 .+7935$ & 4,08307 & $3,0+262$ \\
\hline 11 & , & 55,25 & 950 & 32,132 & $8 \cdot 6,2 ! 5$ & 52,487 & - 7415 & 582,430 & $\cdot 02898$ & 16,87879 & 13,06672 & 10,11563 & 7,83108 & 6,06235 \\
\hline 20 & , & 70.50 & 915 & 28,378 & 891,593 & $6+1,507$ & $\cdot 7995 !$ & 398,251 & .02537 & 10,10363 & 8,07805 & $6,158.5$ & 376 & 1,12811 \\
\hline 21 & - & 52,50 & 815 & 29,730 & 924,323 & 12,787 & .82609 & 482,389 & $\cdot 02657$ & 12,81708 & 10,58806 & $8,7+166.3$ & 7,22550 & 5.96891 \\
\hline 22 & , & 41,25 & 710 & 22,973 & $9.47,296$ & 29,287 & $\cdot 84662$ & 575,682 & .02053 & 11,81875 & 10,00599 & $8,+7121$ & 7,17185 & 6.07176 \\
\hline 23 & , & 39,25 & 7.10 & 22,297 & 969.593 & 29,045 & $\cdot 8665.1$ & 460,320 & $\cdot 01992$ & 9,16957 & 7.91580 & 6.88531 & 5,9 & 5.17017 \\
\hline 24 & , & $2 \cdot, 50$ & 650 & 21,622 & 991.215 & 15,$92 ;$ & .88587 & 642,386 & .01933 & 12,41732 & 11,00013 & $9,7.171$ & 8,63252 & $7,6.1733$ \\
\hline $2 i$ & $\$$ & 15,25 & 550 & 18.919 & 1010,131 & 8,387 & .90278 & 918,088 & $\cdot 01691$ & 15,52185 & 14.01552 & 12,65291 & 11,12272 & 10.31223 \\
\hline 26 & - & 11,50 & $5^{\prime} 0$ & 16,216 & 1026,350 & 5,750 & .91727 & 1001,739 & .01449 & 11,51520 & 13,31436 & 12,21280 & 11,20210 & 10,27560 \\
\hline 27 & . & 8,75 & 590 & 18,243 & 1014,593 & 5,162 & .93357 & 743,898 & .01630 & 12,12554 & 11,32004 & 10.56801 & 9,86595 & $9,210.56$ \\
\hline 28 & , & 18,25 & 640 & 19,595 & 1064188 & 11,680 & .95109 & 184,931 & .01752 & 3,23999 & 3,08152 & 2,83080 & $2,787.16$ & 2,65112 \\
\hline 29 & , & 4,50 & 375 & 16,892 & 1081,080 & 1,687 & .96618 & 628.33 .4 & .01509 & 9,48156 & 9,16089 & 8.8510 .1 & 8,55170 & 8,26212 \\
\hline 30 & , & 1,75 & 240 & 10,811 & $10^{9} 1,891$ & 0,120 & .97584 & 1309,524 & -00966 & 12,65000 & 12,31438 & $12,0.4609$ & $11,75.501$ & 11,47102 \\
\hline 31 & , & 3,75 & 475 & 8,784 & 1100.675 & 1,781 & .98369 & 145.985 & $\cdot 00785$ & $1,1+598$ & 1,12729 & 1,10891 & 1.09082 & 1.07301 \\
\hline 32 & $\bullet$ & 0 & 0 & $18,2+3$ & 1118,918 & 0 & 1 & 0 & 0 & $310,868+3$ & $207,2+365$ & 155,12759 & $12+, 20 \cdot 409$ & 103,81510 \\
\hline & & & & & & & & & & $1_{0}$ & & & & \\
\hline
\end{tabular}


Sia a la superficie del lago, $g$ l'accelerazione di gravità, $\lambda$ un parametro che risulta dalla risoluzione dell'equazione di III grado (corrispondente al caso $m=2$ ); i periodi delle oscillazioni libere si traggono dalla formula :

$$
T=2 \pi a /(g \wedge) 1 / 2
$$

L'equazione che consente la determinazione dei valori di $\lambda$ contiene opportune combinazioni di certe graudezze $I_{0}, I_{1}, I_{2}, I_{3}, I_{4}$, i valori delle quali vengono dedotti dai dati che caratterizzano la forma
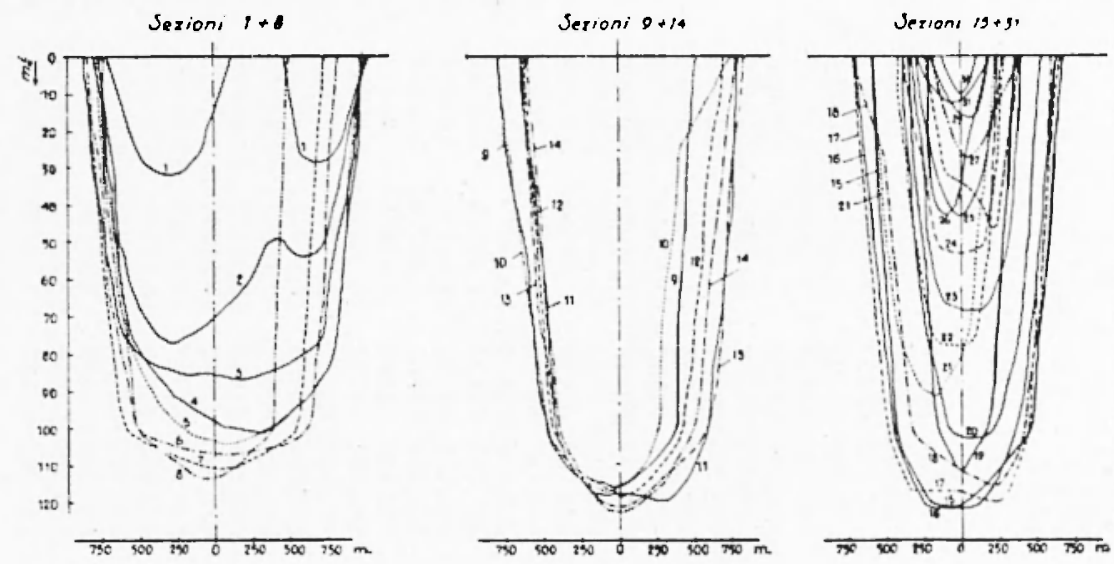

Fig. 2

del lago. Poiché, come si è detto, la curva normale è risultata di forma complessa, la relativa equazione di Chrystal è stata risolta mediante integrazione numerica. Da tale risoluzione (vedi tabella $I$ ), si sono tratti $i$ seguenti valori:

$$
\begin{aligned}
& I_{1}=310,86843 \\
& I_{1}=207,24365 \\
& I_{2}=155,12759 \\
& I_{2}=124,26409 \\
& I_{4}=103,81510
\end{aligned}
$$

Ne è venuta la seguente equazione di III grado in $\lambda$ :

$$
4166,937 \lambda^{3}-63,009 \lambda^{2}+0,16997-0,000095238=0
$$


Il metodo delle approssimazioni successive ha dato i tre seguenti valori per i:

$$
\begin{aligned}
& \lambda_{1}=0.0007677 \\
& \lambda_{2}=0,002515 \\
& \lambda_{3}=0,011839
\end{aligned}
$$

I'oiché $a=0,111892 \mathrm{Mm}^{2}$, conseguono dalla [1] per i tre valori di $i$ ottenuti,

$$
\begin{aligned}
& T_{1}=13^{\mathrm{m}}, 5 \\
& T_{2}=7^{\mathrm{n}}, 5 \\
& T_{33}=3^{\mathrm{m}}, 4
\end{aligned}
$$

the rappresentano i periodi delle sesse uni-li-trinodali del lago di Idro.

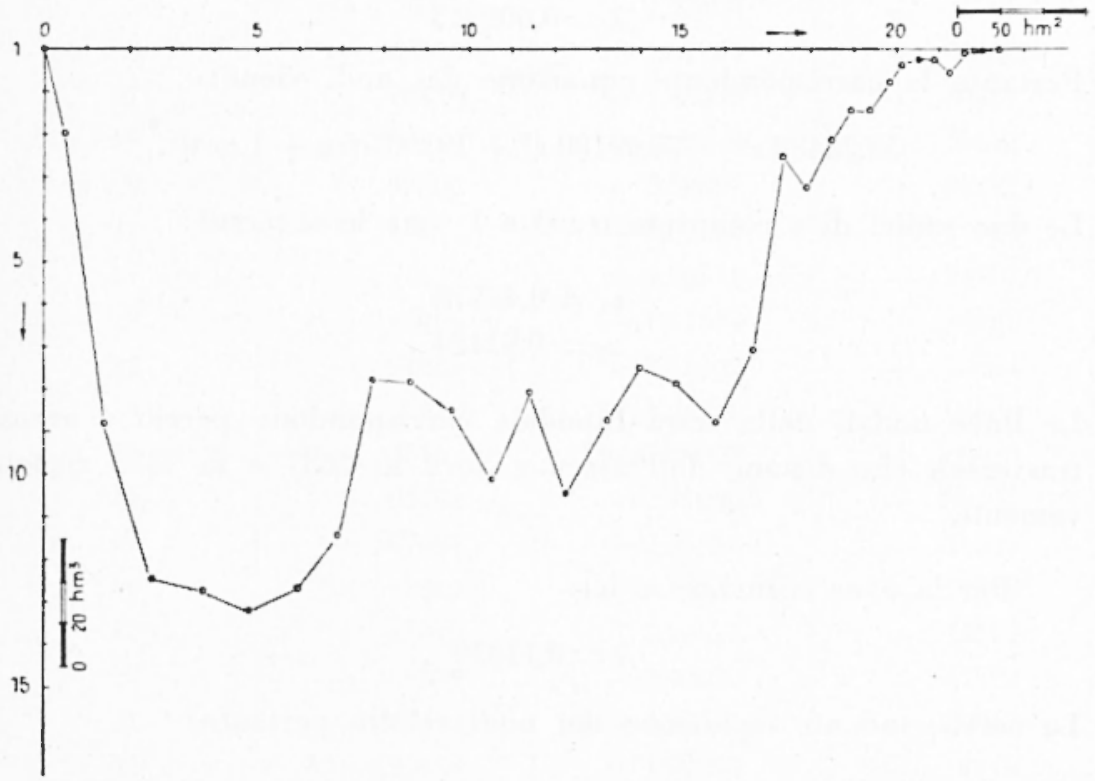

Fig. 3

Per la determinazione dei nodi ra tenuto presente che in essi $d u / d z=0$. Ciò comporta nel caso $m=2$.

$$
4 \frac{A_{1}}{A_{0}} z^{3}+3\left(\frac{A_{4}}{A_{0}}-\frac{A_{2}}{A_{0}}\right) z^{2}+2\left(1-\frac{A}{A_{0}}\right) z=0
$$

dove il valore dei rapporti $A_{2} / A_{0}$ e $A_{2} / A_{0}$ può essere dedotto dal sistema di equazioni in $A_{0}, A_{1}, \ldots$ una volta determinati i valori di $I_{0}, I_{1}, \ldots$ e per ouni valore di $\lambda$. 
Arremo pertanto, con riferimento all'oscillazione libera uninodale l'equazione di III grado in z:

$$
17,40040 z^{3}-17,(12232 z=+5,07+68 z-1=0 .
$$

Risolta tale equazione con il metodo delle approssinazioni successive, si è pervenuti al seguente valore per z.

$$
z=0,71812
$$

che è il solo dei valori di z compresi fra 0 e 1 .

L'uninodo corrisponde quindi ad una sezione che dista dall'estremo Nord m 4890.

Il valore di $\lambda$ corrispondente all'oscillazione libera binodale è:

$$
\lambda=0,002515
$$

Pertanto la corrispondente equazione dei nodi viene:

$$
58,28488 z^{3}-75,69180 z+19,31876 z+1=0 .
$$

Le due radici di z comprese tra 0 e 1 sono le seguenti:

$$
\begin{aligned}
& z_{1}=0,42578 \\
& z^{\prime}=0,91124 .
\end{aligned}
$$

Le linee nodali della sessa hinodale corrispondono perció a sezioni trasversali che distano dall'estremo Nord m 2687 e m 7375 rispettivamente.

Per la sessa trinodale si ha:

$$
\lambda=0,11839 \text {. }
$$

La corrispondente equazione dei nodi risulta pertanto:

$$
10,5896 z^{3}-18,3642 z^{2}+8,9480 z-1=0 \text {. }
$$

Le tre radici di questa equazione sono tutte evidentemente comprese fra 0 e 1. Eccone i valori:

$$
\begin{aligned}
& z_{1}=0,15876 \\
& z^{*}=0,62761 \\
& z^{3}=0,94777
\end{aligned}
$$

A questi valori corrispondono tre sezioni trasversali (trinodi) che distano dall'estremo Nord m 854 , m 4200 , m 8043 rispettivamente. 


\section{Tabelia II}

\begin{tabular}{|c|c|c|c|}
\hline Sxyoni & $I^{0}$ Uninodole & $\mathrm{II}^{0}$ Binodale & IIIO Trinodale \\
\hline 0 & -1 & -1 & -1 \\
\hline 1 & -.911574 & -1.31532 & - .82: 91 \\
\hline 2 & $-.7+170$ & -1.93529 & -.50378 \\
\hline 3 &.$-(i 2: 34)$ & -2.30835 & $-.190(15$ \\
\hline 4 & $-.51331: t$ & -2.357110 & $\therefore-.03(123$ \\
\hline 5 & $-.5 i n u(i . t$ & -2.28400 & $\div-185009$ \\
\hline 6 & -.5153159 & $-1.57 i 5.3$ & +.28050 \\
\hline 7 & -.01170 & -1.453199 & -+.32451 \\
\hline 8 & -.63411 & $-1.112226 i$ & $+.3 \div 621$ \\
\hline$!$ & $-.665 !) k i$ & -0.5833156 & +.32081 \\
\hline 10 & -.69171 & $-11 .(1212010$ & $-1-.29642$ \\
\hline 11 & -.70112 & -11.60 .271 & +.24701 \\
\hline 12 & -. .6s4!19 & $+1.1 .15 \% 7$ & +.18663 \\
\hline $1: 3$ & -.64355 & +1.58202 & : . 1:2694 \\
\hline 11 & -.51518 & +1.95500 & $-.06069 !$ \\
\hline 15 & -.45552 & +2.280416 & $i^{-} .00113$ \\
\hline 16 & -.30763 & -2.450966 & -.05457 \\
\hline 17 & -.17457 & 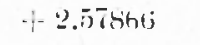 & $-.110 ! 9$ \\
\hline 18 & +.19612 & $1+0.5172 ;$ & -.12773 \\
\hline $1 !$ & -.44012 & $+2.365 t 8$ & $-.1656 i 1$ \\
\hline 201 & +.48559 & +2.15150 & -.17272 \\
\hline 21 & +.97562 & +1.83705 & $-.17(147$ \\
\hline 23 & $-1,2 \cdot 2 \cdot 430$ & +1.52867 & -.16118 \\
\hline 23 & $\div 1.18718$ & $-1.17(155$ & -.14525 \\
\hline 21 & $+1,76280$ & -0.76709 & -.12302 \\
\hline 25 & $\because-2,112164$ & $\div 0.96471$ & -.05742 \\
\hline 26 & $-2,2568.1$ & -0.11147 & $-.070 s 0$ \\
\hline 27 & $-2,536668$ & -0.18371 & -.03551 \\
\hline 28 & $-+2,855 ! 15$ & $-1 .(149)=8$ & +.00920 \\
\hline .29 & $+3,145$ & -1.57598 & +.01348 \\
\hline 30 & $\therefore-3,341835$ & -1.93497 & +.08472 \\
\hline 31 & $\div 3, \overline{5} 0 \cdot 9$ & $-2.2401 i$ & +.11186 \\
\hline 33 & $+3,85: 76$ & -2.91181 & +.17340 \\
\hline
\end{tabular}




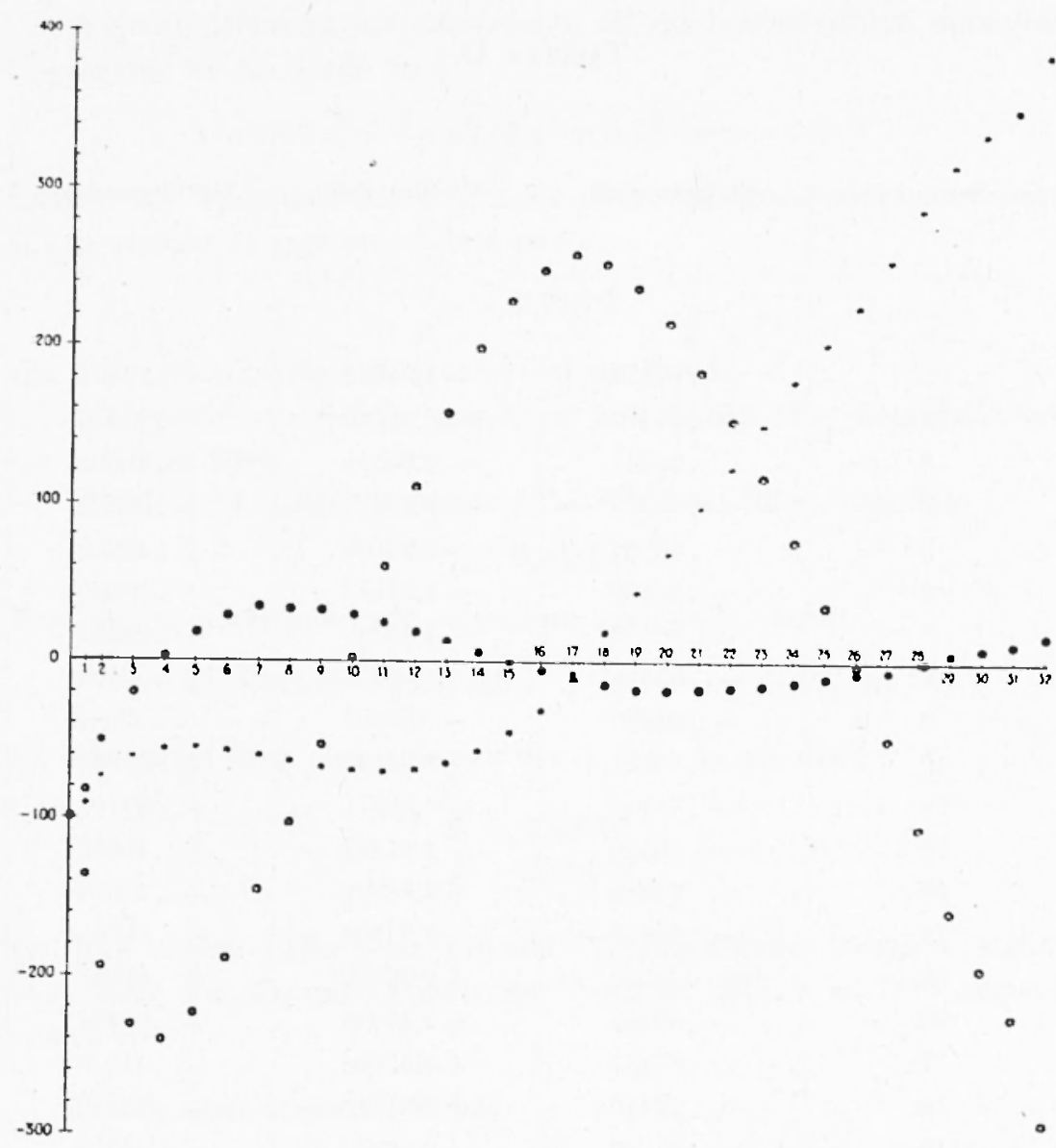

Fig. 4

Fu calcolato poi l'andamento dello spostamento verticale della superficie del lago, corrispondentemente alle tre oscillazioni libere studiate.

Le equazioni che danno l'andamento degli spostamenti verticali sollo:

$$
\begin{gathered}
\zeta^{\prime}=17,40040 z^{3}-17,62232 z^{2}+5,07468 z-1 \\
\text { per la sessa uninodale } \\
\zeta^{\prime \prime}=58,28438 z^{3}-75,69180 z^{2}+19,31876 z+1 \\
\text { per la sessa hinodale } \\
\zeta^{\prime \prime \prime}=10,5896 z^{3}-18,3642 z^{2}+\begin{array}{c}
8,9480 z-1 \\
\text { per la sessa trinodale }
\end{array}
\end{gathered}
$$




\begin{tabular}{|c|c|c|}
\hline \multirow{4}{*}{$\begin{array}{l}\omega \\
\lrcorner \\
\varangle \\
0 \\
0 \\
z \\
\frac{m}{m}\end{array}$} & $\stackrel{\circ}{\stackrel{0}{*}}$ & 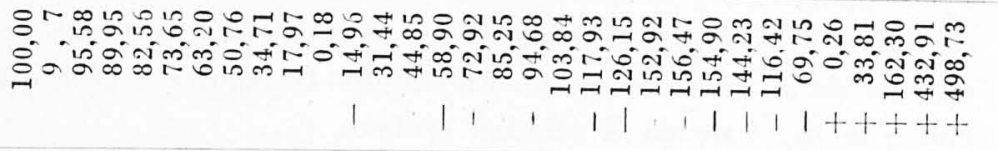 \\
\hline & $\stackrel{0}{\tilde{z}} \overline{0}$ & 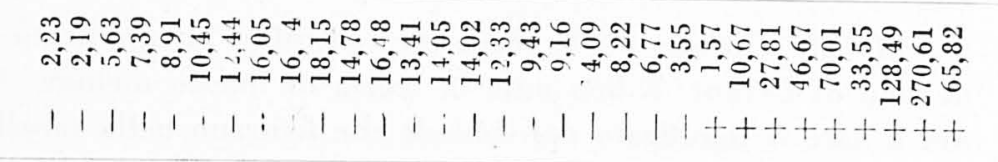 \\
\hline & is & 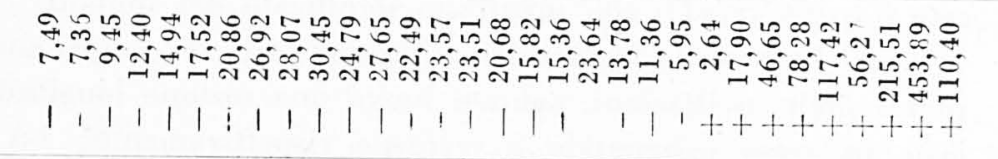 \\
\hline & 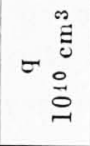 & 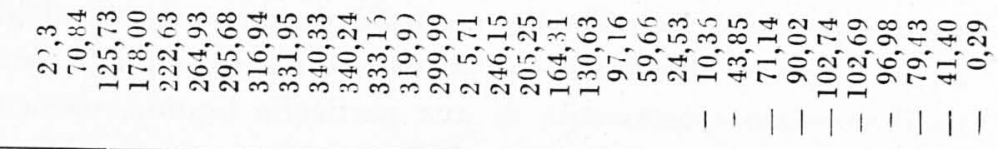 \\
\hline
\end{tabular}

ํㅜㅇ ๓ $\therefore \equiv$ ह

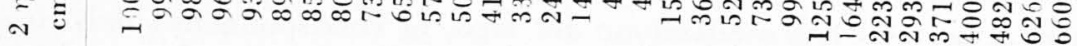

$\underset{\sim}{\stackrel{0}{\Xi} \Xi}$

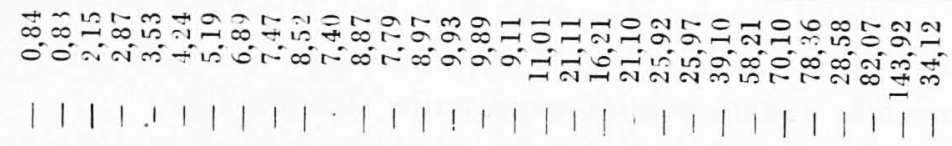

W.

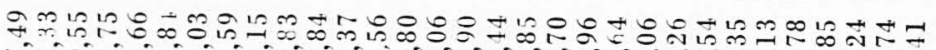

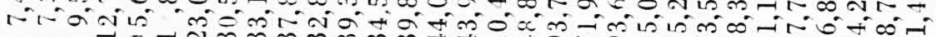
จ $\stackrel{0}{0}$

0

0

$=\frac{\substack{0 \\ 0}}{0}$

๓ जिड

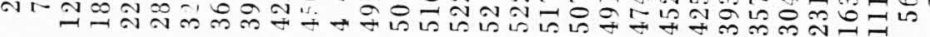

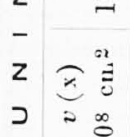

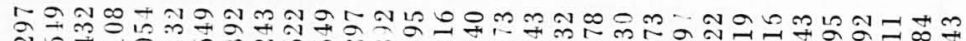
बิ o A

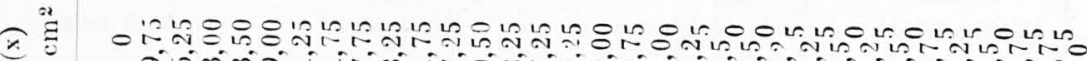
ลิง

in

$$
\begin{aligned}
& \text { ह } \\
& \langle\stackrel{0}{0} \text { in: }
\end{aligned}
$$

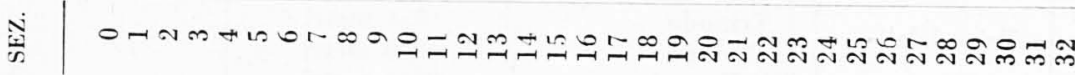


Preso uguale ad 1 lo spostamento iniziale all'estremo Nord, sono stati calcolati, di sezione in sezione, gli spostamenti verticali relativi ad ognuma delle tre sesse cilate. La tabella lI riporta i risultati dei calcoli e la fig. + ne dí il relativo grafico.

Metodo Defant. - A scopo di controllo, ho applicato anche il metodo di Defant. is ben nota la teoria di questo metodo. Mi limito qui a dare il significato dei simboli che figurano nella tabella III. In essa $\grave{e} a=4 \pi^{-j} g T^{2}$, con manifesto significato dei simboli; $\backslash(x)$ rap,presenta l’intervallo fra sezione e sezione; 2 ミn. 2 lju esprimono le ampiezze delle oscillazioni, contate lungro una sezione longitudinale del lago, in senso orizzontale e verticale rispetlivamente; $2 \mathrm{~J}$. u da la variazione dello spostamento verticale da una sezione allalira; $q=$ $2 \eta_{0} v(x)$ eprime il volume dellacqua che nel tempo $T / 4$, fra il riposo ed il massimo spostamento di una particella liquida, passa attraverso una generica sezione $S(x)$.

Quando il periodo $T$ dell'oscillazione libera considerata coincide con il periodo dell'oscillazione del lago, in corrispondenza dell'ultima sezione si lovrà avere $q=0$.

Per la sessa uninodale, il valore $T=1.4^{m}, 4$ ha portato al residuo $q=142,03: 10^{10} \mathrm{~cm}^{3}$. Dopo un altro tentativo per $T=12^{\mathrm{m}, 2}$ il residuo è stato praticamente nullo (tabella IIJ).

Per quanto conceme la sessa binodale un residuo praticamento nullo si è avuto con il periodo $T=7^{\mathrm{m}, 5}$ (tal)ella Ill).

La fig. 5 da la posizione dei nodi e l'andamento degli spostanenti lungo il lago, per le due oscillazioni libere considerate, ottenute con il metodo di Defant.

La ricerca analitica condolla sul lago di Idro ha portato ai risultati che qui ora riassumo

\begin{tabular}{|c|c|c|c|}
\hline \multicolumn{2}{|c|}{ Oscillazioni libere } & $\begin{array}{l}\text { Primo metodo } \\
\text { (Hidaka) }\end{array}$ & $\begin{array}{l}\text { Secondo metodo } \\
\text { (Defant) }\end{array}$ \\
\hline Uninodale & $\left\{\begin{array}{l}\text { periodo } \\
\text { nodo dall'estremo } \\
\text { Nord }\end{array}\right.$ & $\begin{array}{c}13,5 \\
4890 \text { metri }\end{array}$ & $\begin{array}{l}12,2 \\
1650 \text { metri }\end{array}$ \\
\hline Binodale & $\begin{array}{l}\text { ( periodo } \\
\text { I Binodo } \\
\text { I II Binodo }\end{array}$ & $\begin{array}{l}7,5 \\
2687 \text { metri } \\
7375 \text { metri }\end{array}$ & $\begin{array}{c}7,5 \\
2700 \text { metri } \\
7800 \text { metıi }\end{array}$ \\
\hline Trinodale & $\left\{\begin{array}{l}\text { periodo } \\
\text { I Trinodo } \\
\text { II Trinodo } \\
\text { III Trinodo }\end{array}\right.$ & $\begin{array}{c}3,4 \\
854 \text { metri } \\
4200 \text { metri } \\
80.13 \text { metri }\end{array}$ & \\
\hline
\end{tabular}




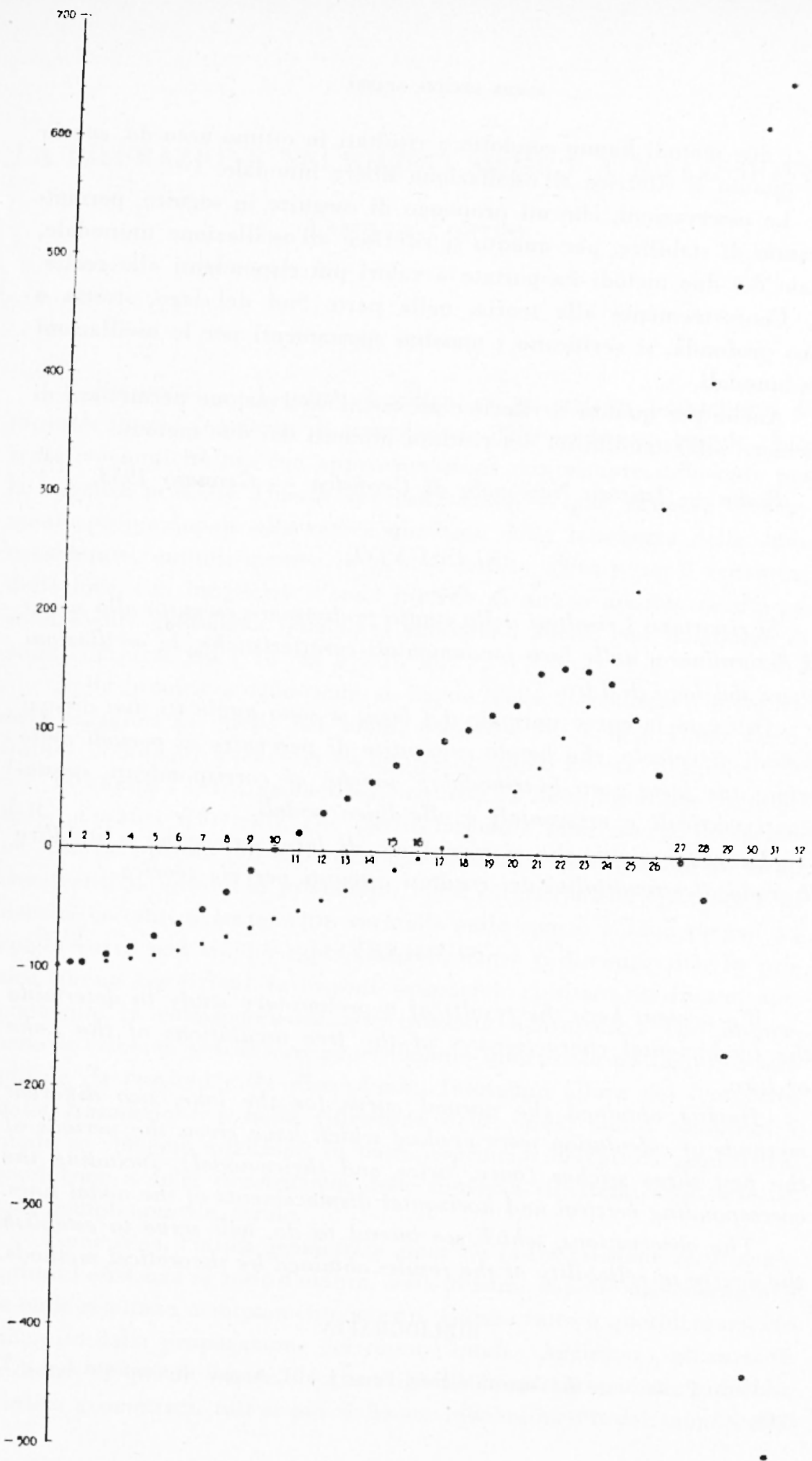

Fig. 5 
I due metodi hanno condotto a risultati in ottimo accordo, specie per quanto si riferisce all'oscillazione libera binodale.

Le osservazioni, che mi propongo di eseguire in seguito, permetteranno di stabilire, per quanto si riferisce all'oscillazione uninodale, quale dei due metodi ha portato a valori più rispondenti alla realtà.

Conformemente alla teoria, nella parte Sud del lago, stretta e poco profonda, si verificano i massimi spostamenti per le oscillazioni uni-binodali.

Anche per quanto si riferisce ad essi, l'osservazione permetterà di decidere sull'attendibilità dei risultati ottenuti dai due metodi.

Roma - Istituto Nazionale di Geofisica - Gennaio 1953.

\section{RIASSUINTO}

Si riportano $i$ risultati dello studio preliminare eseguito allo scopo di determinare, nelle loro fondamentali caratteristiche, le oscillazioni libere del lago di Idro.

Ottenuta la curva normale del lago, si sono applicati due diversi metodi di calcolo, che hanno consentito di pervenire ai periodi delle prime tre sesse (uni,-bi-trinodale) nonché ai corrispondenti spostamenti verticali e orizzontali e alle linee nodali.

Le osservazioni, che ci riserviamo di fare, serviranno a stabilire il grado di attendibilità dei risultati ottenuti per via teorica.

\section{SLMIIARY}

We present here the results of a proliminary study to determine the fundamental characteristics of the free oscillations of the Lalie of Idro.

Having obtained the normal curve for the lake, two different methods of calculation uere applied which have given the periods of the first three seiches (once, twice and thrice-nodal), including the corresponding vertical and horizontal displacements of the nodal lines.

The observations, which we intend to do, will serve to establish the degree of reliability of the results obtained by theoretical methods.

\section{BIBLIOGRAFIA}

Caror P., Le sesse del lago di Gardr. Parte I e II, Anmali di Geofisica I, 1 e 2, 19.48. 